\title{
Targeted photodynamic therapy for prostate cancer: Inducing apoptosis via activation of the caspase-8/-3 cascade pathway
}

\author{
TIANCHENG LIU ${ }^{1}$, LISA Y. WU ${ }^{1}$, JOSEPH K. CHOI ${ }^{1}$ and CLIFFORD E. BERKMAN ${ }^{1,2}$ \\ ${ }^{1}$ Department of Chemistry, Washington State University, Pullman, WA 99164-4630; \\ ${ }^{2}$ Cancer Targeted Technology, Woodinville, WA 98072, USA
}

Received November 9, 2009; Accepted December 29, 2009

DOI: 10.3892/ijo_00000553

\begin{abstract}
The limitation of specific delivery of photosensitizers to tumor sites, represents a significant shortcoming of photodynamic therapy (PDT) application at present. Prostate-specific membrane antigen (PSMA), a validated biomarker for prostate cancer, has attracted considerable attention as a target for imaging and therapeutic applications for prostate cancer. The present study focuses on the investigation of a PSMA inhibitorconjugate of pyropheophorbide-a (Ppa-conjugate 2.1) for a targeted PDT application and the mechanism of its mediatedcell death in prostate cancer cells. Multiple fluorescence labeling methods were employed to monitor PDT-treated prostate cancer cells by confocal laser scanning microscopy. Our results demonstrate that Ppa-conjugate 2.1 mediated apoptosis is specific to PSMA ${ }^{+}$(positive) LNCaP cells, but not PSMA- (negative) PC-3 cells. Furthermore, these results indicate that following PDT, the activation of caspase- $8,-3,-9$, cleavage of poly(ADP-ribose) polymerase (PARP) and DNA fragmentation is sequential. The appearance of cleaved $B$-actin further supported involvement of caspase-3. Specific caspase inhibitor blocking studies reveal that the caspase-8/-3 cascade pathway plays a key role in apoptosis of $\mathrm{LNCaP}$ cells induced by Ppa-conjugate 2.1. The demonstrated selective targeting and efficacy of this agent suggests that targeted PDT could serve as an alternative treatment option for prostate cancer.
\end{abstract}

\section{Introduction}

Photodynamic therapy (PDT) has emerged as a minimally invasive regimen for the treatment of cancers and pre- or non-cancerous conditions and as such, offers an attractive alternative or complement to conventional therapies (1-3). The therapeutic action of PDT is based on the generation of

Correspondence to: Professor Clifford E. Berkman, Department of Chemistry, Washington State University, Pullman, WA 99164-4630, USA

E-mail: cberkman@wsu.edu

Key words: prostate cancer, apoptosis, caspase- 8 reactive oxygen species (ROS) that are formed upon light activation of a photosensitizer (PS) such as a porphyrinic pigment. The resulting excited PS transfers its energy to molecular oxygen in tissue to generate ROS. Singlet oxygen is assumed to be the key cytotoxic ROS responsible for localized oxidative cell damage and death $(1,3,4)$. However, PDT is not yet an integral part of clinical practice due, in part, to the lack of selectivity for target cells and concomitant potential for systemic damage. To transform PDT into a clinically relevant treatment option for prostate cancer will require a considerably greater selectivity in the targeting of the PS to tumor cells in order to limit photosensitivity of nontarget tissues while enhancing drug accumulation in tumors.

In light of the present lack in the effective targeting of PSs for PDT, we have focused on developing a method for the targeted delivery of PSs for the selective abrogation of prostate cancer cells $(5,6)$. Specifically, we have designed chemical agents that exhibit high-affinity and specificity for the prostate cancer enzyme-biomarker prostate-specific membrane antigen (PSMA) (5). PSMA is a type II cell-surface glycoprotein predominantly restricted to prostatic tissue and is strongly expressed on prostate tumor cells (7). Expression levels increase with disease progression, being highest in metastatic disease, hormone refractory cancers, and highergrade lesions $(8,9)$. Endothelial-expression of PSMA in the neovasculature of a variety of non-prostatic solid malignancies has also been detected. Therefore, it is not surprising that PSMA has attracted considerable attention as a biomarker and target for the delivery of imaging and therapeutic agents.

PDT-induced apoptosis usually proceeds through two main pathways (10). The first, referred to as the extrinsic or cytoplasmic pathway, is initiated by death receptors (DRs) of the tumor necrosis factor receptor (TNFR) superfamily that subsequently activate caspase- 8 , which in turn activates downstream caspases. There are few photosensitizers, and with limited cell types, have been involved in the activation of this pathway following PDT experiments (11-13). The second pathway is the intrinsic or mitochondrial pathway. In response to PDT treatment, the outer mitochondrial membrane becomes permeable leading to the release of cytochrome-c into the cytosol where it interacts with apoptotic protease activating factor 1 (Apaf-1) and dATP forming the apoptosome. This is followed by the activation of caspase-9, which then activates caspase-3. Caspase- 3 subsequently activates the remainder of the caspase cascade, cleaves PARP, finally 
leading to apoptosis (10). The subcellular localization of photosensitizes in cells is a determining factor for the initiation of different apoptotic pathways (14). Most photosensitizes are not tumor cell-specific and being lipophilic, preferentially localize in the intracellular membrane systems, particularly mitochondria. Therefore, it is not surprising that most nontargeted PDT-mediated apoptosis proceeds through the intrinsic pathway (15).

We previously reported that phosphoramidate peptidomimetic PSMA inhibitors were capable of both cell-surface labeling of prostate cancer cells and intracellular delivery for PDT $(5,6)$. Compared with the photosensitizer conjugate from our earlier studies (6), herein we describe the conjugation of a new peptidomimetic inhibitor core $\mathbf{1 . 1}$ to the photosensitizer pyropheophorbide-a (Ppa) (Fig. 1). In addition, we reveal the capability of this construct for increased affinity to purified PSMA and selective induction of cellular apoptosis in prostate cancer cells in vitro at a lower concentration. Following PDT experiments, cytotoxicity $\left(\mathrm{LC}_{50}\right)$ for the PSMA inhibitorphotosensitizer conjugate was determined by the MTT assay. Evidence for apoptosis was confirmed by nuclear staining, poly(ADP-ribose) polymerase (PARP) p85 fragment immunofluorescence, Western blotting for the caspase-3-cleaved $\beta$-actin, and the TUNEL assay. Involvement and the initiation sequence of caspase cascade pathway were investigated using specific fluorescent or non-fluorescent caspase inhibitors in PDT-treated cells. Fluorescence images of cells were obtained using confocal laser scanning microscopy.

\section{Materials and methods}

Cell lines, reagents, and general procedures. LNCaP and PC-3 cells were obtained from the American Type Culture Collection (Manassas, VA). The mouse monoclonal anti-cytokeratin 8 antibody, rabbit polyclonal anti-PARP p85 antibody and goat anti-rabbit IgG-FITC were obtained from Sigma-Aldrich (St. Louis, MO). Rabbit polyclonal anti-human Fractin (caspasecleaved actin) was obtained from BD Biosciences (San Jose, CA). Normal goat serum was obtained from BioGenex (San Ramon, CA). The CaspGLOW ${ }^{\text {тм }}$ fluorescein active caspases $-8,-3$ and -9 staining kits and irreversible inhibitors (Z-IETDFMK, Z-DEVD-FMK and Z-LEHD-FMK) of caspases -8, -3 and -9 were obtained from BioVision Inc. (Mountain View, CA). 4',6-diamidino-2-phenylindol dihydrochloride (DAPI) and Hoechst 33342 (Hoe33342) were obtained from InvitrogenMolecular Probes. DeadEnd Fluorometric TUNEL System was obtained from Promega Corp. (Madison, WI). Pyropheophorbide-a (Ppa) was obtained from Frontier Scientific, Inc. (Logan, UT). All other chemicals and cell-culture reagents were purchased from Fisher Scientific (Sommerville, NJ), Pierce (Rockford, IL), or Sigma-Aldrich. All solvents used in chemical reactions were anhydrous and obtained as such from commercial sources. All other reagents were used as supplied unless otherwise stated. ${ }^{1} \mathrm{H},{ }^{13} \mathrm{C}$, and ${ }^{31} \mathrm{P}$ NMR spectra were recorded on a Bruker DRX $300 \mathrm{MHz}$ NMR Spectrometer. ${ }^{1} \mathrm{H}$ NMR chemical shifts are relative to TMS $(\delta=0.00 \mathrm{ppm}), \mathrm{CDCl}_{3}(\delta=7.26 \mathrm{ppm}) .{ }^{13} \mathrm{C} \mathrm{NMR}$ chemical shifts are relative to $\mathrm{CDCl}_{3}(\delta=77.23 \mathrm{ppm}) .{ }^{31} \mathrm{P} \mathrm{NMR}$ chemical shifts in $\mathrm{CDCl}_{3}$ was externally referenced to $85 \%$ $\mathrm{H}_{3} \mathrm{PO}_{4}(\delta=0.00 \mathrm{ppm})$ in $\mathrm{CDCl}_{3}$.
Preparation of Ppa-conjugate 2.1. The NHS ester of pyropheophorbide-a (Ppa-NHS) was prepared as previously described in our previous work $(5,6)$. A solution of Ppa-NHS ester $(6 \mu \mathrm{mol})$ in $100 \mu \mathrm{l}$ DMSO was added to a stirred solution of the inhibitor core $1.1\left(2 \mu \mathrm{mol}, 100 \mu \mathrm{l}\right.$ of $20 \mathrm{mM}$ in $\left.\mathrm{H}_{2} \mathrm{O}\right), 160 \mu \mathrm{l} \mathrm{H}_{2} \mathrm{O}$, and $40 \mu \mathrm{l}$ of $1 \mathrm{M} \mathrm{NaHCO}_{3}$. The reaction mixture was stirred for $6 \mathrm{~h}$ in the dark at room temperature. The $\mathrm{pH}$ of the resulting solution was then adjusted to 9.3 by the addition of $8 \mu \mathrm{l}$ of $1 \mathrm{M} \mathrm{Na}_{2} \mathrm{CO}_{3}$. The unreacted inhibitor core $\mathbf{1 . 1}$ was scavenged by stirring with $25 \mathrm{mg}$ of Si-Isocyanate resin (SiliCycle, Inc., Quebec, Canada) overnight at room temperature. The solution was subsequently centrifuged $(9,000 \mathrm{rpm}, 10 \mathrm{~min})$ and the supernatant was lyophilized in a $2-\mathrm{ml}$ microcentrifuge tube. Unreacted and/or hydrolyzed Ppa-NHS was removed by successively triturating the lyophilized solid with $1 \mathrm{ml}$ portions of DMSO and centrifuging the mixture (1 $\mathrm{min}$ at $13,000 \mathrm{rpm}$ ) after each wash; this process was repeated 10 times. The Ppa-conjugated inhibitor $\mathbf{2 . 1}$ was dissolved in $50 \mathrm{mM}$ Tris buffer ( $\mathrm{pH} 7.5$ ) to give a final concentration of $2 \mathrm{mM}(\sim 800 \mu \mathrm{l})$.

$I C_{50}$ determination for inhibitor core 1.1 and Ppa-conjugate 2.1. Inhibition studies were performed as described previously with only minor modifications (16-18). Working solutions of the substrate N-[4-(phenylazo)-benzoyl]-glutamyl- $\gamma$ glutamic acid (PABGgG) and inhibitor were made in Tris buffer (50 mM, pH 7.4). Working solutions of purified PSMA were diluted in Tris buffer $(50 \mathrm{mM}, \mathrm{pH} 7.4$ containing $1 \%$ Triton X-100) to provide from 15 to $20 \%$ conversion of substrate to product in the absence of inhibitor. A typical incubation mixture (final volume $250 \mu \mathrm{l}$ ) was prepared by the addition of either $25 \mu \mathrm{l}$ of an inhibitor solution or $25 \mu \mathrm{l}$ Tris buffer (50 mM, pH 7.4) to $175 \mu 1$ Tris buffer (50 mM, pH 7.4 containing $1 \%$ Triton X-100) in a test tube. PABGgG $(25 \mu \mathrm{l}, 10 \mu \mathrm{M})$ was added to the above solution. The enzymatic reaction was initiated by the addition of $25 \mu \mathrm{l}$ of the PSMA working solution. In all cases, the final concentration of PABGgG was $1 \mu \mathrm{M}$ while the enzyme was incubated with five serially diluted inhibitor concentrations providing a range of inhibition from 10 to $90 \%$. The reaction was allowed to proceed for $15 \mathrm{~min}$ with constant shaking at $37^{\circ} \mathrm{C}$ and was terminated by the addition of $25 \mu 1$ methanolic TFA (2\% trifluoroacetic acid by volume in methanol) followed by vortexing. The quenched incubation mixture was quickly buffered by the addition of $25 \mu 1 \mathrm{~K}_{2} \mathrm{HPO}_{4}(0.1 \mathrm{M})$, vortexed, and centrifuged $(10 \mathrm{~min}$ at 7,000 g). An $85-\mu 1$ aliquot of the resulting supernatant was subsequently quantified by HPLC as previously described. $\mathrm{IC}_{50}$ values were calculated using KaleidaGraph 3.6 (Synergy software).

Cell culture. LNCaP (PSMA-positive; PSMA+) and PC-3 (PSMA-negative; PSMA-) cells were grown in T-75 flasks with complete growth medium [RPMI-1640 containing 10\% heat-inactivated fetal calf serum (FBS), $100 \mathrm{U}$ of penicillin and $100 \mu \mathrm{g} / \mathrm{ml}$ streptomycin] in a humidified incubator at $37^{\circ} \mathrm{C}$ and $5 \% \mathrm{CO}_{2}$. Confluent cells were detached with a $0.25 \%$ trypsin $0.53 \mathrm{mM}$ EDTA solution, harvested, and plated in 2-well slide chambers at a density of $4 \times 10^{4}$ cells/well. Cells were grown for 3 days before conducting the following experiments (6). 
In vitro PDT with Ppa-conjugate 2.1. $\mathrm{LNCaP}$ and $\mathrm{PC}-3$ cells grown in 2-well slide chambers for 3 days were washed twice in $37^{\circ} \mathrm{C}$ pre-warmed medium A (phosphate-free RPMI-1640 containing $1 \% \mathrm{FBS}$ ), and then incubated with $1 \mathrm{ml}$ of Ppaconjugate $2.1(1 \mu \mathrm{M})$ in pre-warmed medium A for $2.5 \mathrm{~h}$ in a humidified incubator at $37^{\circ} \mathrm{C}$ and $5 \% \mathrm{CO}_{2}$, which allowed internalization of Ppa-conjugate 2.1 bound to PSMA to occur. Cells treated with Ppa-conjugate 2.1 were washed in the $37^{\circ} \mathrm{C}$ pre-warmed phenol-free medium RPMI-1640 once, and then irradiated with white light $\left(7.5 \mathrm{~J} / \mathrm{cm}^{2}\right.$, with $25 \mathrm{~mW} / \mathrm{cm}^{2}$ fluence rate) for $10 \mathrm{~min}$ in pre-warmed phenol-free RPMI-1640. The light source was a $100-\mathrm{W}$ halogen lamp, which was filtered through a $10-\mathrm{cm}$ column of water, and then filtered through a Lee Primary Red filter (Vincent Lighting) to remove light with wavelengths $<600 \mathrm{~nm}$.

TUNEL staining in situ. PDT-treated cells were placed in pre-warmed complete growth medium, and returned to a humidified incubator at $37^{\circ} \mathrm{C}$ and $5 \% \mathrm{CO}_{2}$ for $16 \mathrm{~h}$ and allowed to recover. Cells were rinsed twice in ice-cold PBS (phosphate-buffered saline), and then fixed with $4 \%$ paraformaldehyde in PBS for $15 \mathrm{~min}$ at room temperature. The fixed cells were washed twice in ice-cold PBS, permeabilized in $0.2 \%$ Triton X-100 solution in PBS for 5 min at room temperature, and rinsed twice in PBS for 5 min at room temperature. The TUNEL (terminal deoxynucleotidyl transferase dUTP nick-end labeling) assay was performed according to the manufacturer's instructions. All treated cells were counterstained with DAPI and mounted in Vectashield ${ }^{\circledR}$ Mounting Medium (Vector-Laboratories) for microscopy.

Cell viability assay. CellTiter $96^{\circledR}$ Non-Radioactive Cell Proliferation Assay (Promega Corp.) was employed in this experiment. Following PDT treatment with Ppa-conjugate 2.1 at $0,0.25,0.5,0.75,1$, and $1.25 \mu \mathrm{M}$, cells were incubated with pre-warmed normal growth medium, and allowed to recover for $16 \mathrm{~h}$ in a humidified incubator at $37^{\circ} \mathrm{C}$ and $5 \%$ $\mathrm{CO}_{2}$. After removal of the old cell media, the treated cells were replaced in freshly prepared dye solution (150 $\mu \mathrm{l}$ Dye/ $500 \mu \mathrm{l}$ of fresh normal growth medium), and incubated at $37^{\circ} \mathrm{C}$ for up to $4 \mathrm{~h}$ in a humidified, $5 \% \mathrm{CO}_{2}$ incubator. After incubation, $1 \mathrm{ml}$ of the solubilization solution was added to each well, mixed gently, and the slides were allowed to stand for $2.5 \mathrm{~h}$ in a $5 \% \mathrm{CO}_{2}, 37^{\circ} \mathrm{C}$ incubator to completely solubilize the formazan crystals. By mixing gently the final solution, before three equal $200 \mu \mathrm{l}$ of each sample were transferred into 3-wells of 96-well plate, respectively. Only dye solution and solubilization (without cells) as blank, cells (no inhibitor) as control. The absorbance was detected and recorded at $570 \mathrm{~nm}$ wavelength using FLUOstar Omega (BMG Labtech Inc.). A graph was created from average absorbance value of each sample by excel 2003, with Cell Viability (\%) via Ppa-conjugate 2.1 concentration.

In situ labeling of active caspases. Following PDT treatment with Ppa-conjugate $\mathbf{2 . 1}$ at $1 \mu \mathrm{M}$, cells were incubated with $1 \mathrm{ml}$ of pre-warmed phosphate-free RPMI-1640 containing $10 \%$ FBS, and allowed to recover for varied times (1, 2 and $4 \mathrm{~h}$ ) in a humidified incubator at $37^{\circ} \mathrm{C}$ and $5 \% \mathrm{CO}_{2}$, by the end of the last $30 \mathrm{~min}$, replaced with $500 \mu \mathrm{l}$ of pre-warmed phosphate-free RPMI-1640 containing 10\% FBS, $1.7 \mu \mathrm{l}$ of each fluorescent inhibitor of caspases $-8,-3,-9$ and $5 \mu \mathrm{g} / \mathrm{ml}$ of Hoechst 33342 at $37^{\circ} \mathrm{C}$ incubator, according to the manufacturer's manual. The treated-cells were washed twice in washing buffer (BioVision Inc.), once in ice-cold KRB buffer pH 7.4 (mM: NaCl 154.0, KCl 5.0, $\mathrm{CaCl}_{2}$ 2.0, $\mathrm{MgCl}_{2}$ 1.0, HEPES 5.0, D-glucose 5.0), and then fixed in freshly prepared $4 \%$ formaldehyde in KRB for $15 \mathrm{~min}$, following by neutralized for $10 \mathrm{~min}$ in $50 \mathrm{mM}$ Tris buffer $\mathrm{pH} 7.5$ at room temperature and finally mounted in Vectashield Mounting Medium for microscopy.

Inhibition of caspases. LNCaP cells were incubated for $2.5 \mathrm{~h}$ at $37^{\circ} \mathrm{C}$ with $1 \mathrm{ml}$ of Ppa-conjugate $2.1(1 \mu \mathrm{M})$ and caspase inhibitor $(10 \mu \mathrm{M})$ in pre-warmed medium A. The treated cells were subjected to light exposure under the same condition described above. The PDT-treated cells were placed in $1 \mathrm{ml}$ of caspase inhibitor $(10 \mu \mathrm{M})$ in pre-warmed phosphate-free RPMI-1640 containing 10\% FBS, and allowed to recover for $1.5 \mathrm{~h}$ (active caspase assay) or $16 \mathrm{~h}$ (TUNEL staining) in a humidified incubator at $37^{\circ} \mathrm{C}$ and $5 \% \mathrm{CO}_{2}$. For active caspase assay, the treated cells were replaced with $500 \mu \mathrm{l}$ of pre-warmed phosphate-free RPMI-1640 containing 10\% FBS, $1.7 \mu \mathrm{l}$ of each fluorescent inhibitor of caspases $-8,-3$ and -9 , respectively (according to the manufacturer's manual) and $5 \mu \mathrm{g} / \mathrm{ml}$ of $\mathrm{HOE} 33342$ at $37^{\circ} \mathrm{C}$ incubator for $30 \mathrm{~min}$ prior to cell fixation. For TUNEL labeling, we follow the protocol described above.

Detection of PARP p85 fragment. Following PDT treatment, cells were incubated with pre-warmed complete growth medium, and allowed to recover for varied times $(2,4,6$ and $8 \mathrm{~h}$ ) in a humidified incubator at $37^{\circ} \mathrm{C}$ and $5 \% \mathrm{CO}_{2}$. Cells were firstly rinsed twice in ice-cold PBS, then fixed and permeabilized as described in the protocol (TUNEL staining in situ). Cells were then blocked for $2 \mathrm{~h}$ in blocking buffer ( $0.1 \%$ Tween-20 with $5 \%$ goat normal serum in PBS) at room temperature, rinsed once in PBS, incubated with the anti-PARP p85 fragment antibody (1:100) in blocking buffer at $4^{\circ} \mathrm{C}$ overnight, then successively washed twice in PBS, $0.1 \%$ Tween-20 in PBS, and finally in PBS for $10 \mathrm{~min}$ at room temperature. Finally, cells were incubated with the fluorescein conjugate of goat anti-rabbit antibody (1:40) in $1 \% \mathrm{BSA}$ in PBS for $2 \mathrm{~h}$ at room temperature, counterstained with DAPI, and then mounted in Vectashield Mounting Medium for microscopy (6).

Confocal laser scanning microscopy. Cells were visualized under x40 oil immersion objective using a LSM 510 META Laser Scanning Microscope. DAPI or Hoe33342 was excited with a Diode Laser (405 nm), and the emission collected with a BP420-480 nm filter. Fluorescein dye staining active caspases $-8,-3$ and -9 were excited using $488 \mathrm{~nm}$ from an Argon Laser, and the emission collected with a LP505 nm filter. To reduce interchannel cross-talk, a multi-tracking technique was used, and images were taken at a resolution of 1,024x1,024 pixels. Confocal scanning parameters were set up so that the control cells without treatment had no fluorescent signal from background. The pictures were edited 


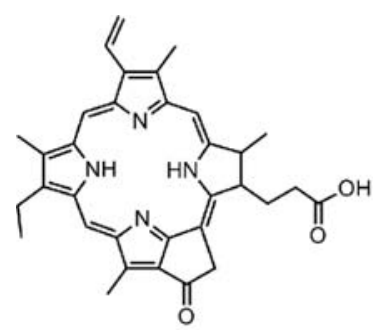<smiles>NCCCCCC(=O)NC(COP(=O)(O)NC(CCC(=O)O)C(=O)O)C(=O)O</smiles>

PSMA inhibitor core 1.1

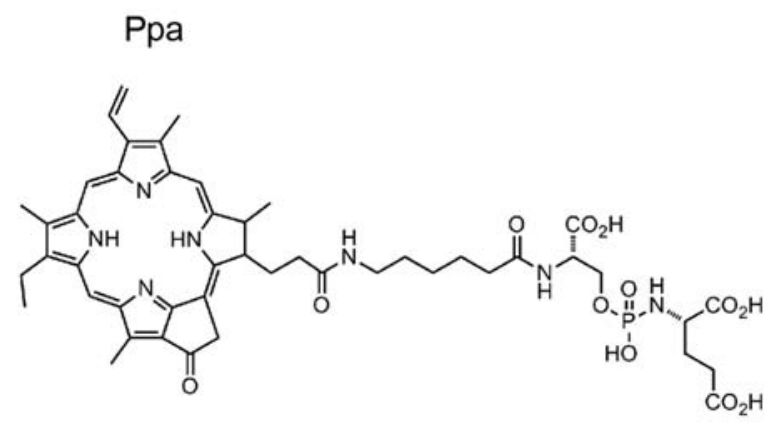

Ppa-conjugate 2.1

Figure 1. Structures of phosphoramidate peptidomimetic inhibitor core 1.1, Ppa, and its Ppa-conjugate 2.1.

by National Institutes of Health (NIH) Image J software (http://rsb.info.nih.gov/ij) and Adobe Photoshop CS2.

Whole cell lysate extraction and Western blotting. At 2 h-post PDT, the control (inhibitor treatment, without light irradiation) and PDT-treated LNCaP cells were collected by scraping, washed once in ice-cold PBS and treated with 3-folded volumes of lysis buffer (1\% NP-40, $20 \mathrm{mM}$ Tris $\mathrm{pH} 8.0$, $137 \mathrm{mM} \mathrm{NaCl}, 10 \%$ glycerol) supplemented with $1 \mathrm{X}$ antiprotease cocktail for $15 \mathrm{~min}$ on ice, and transferred to Eppendorf tubes for centrifugation at $10,000 \mathrm{~g}$ for $15 \mathrm{~min}$ at $4^{\circ} \mathrm{C}$. Protein concentrations were determined using BCA. Western blotting was performed as described previously with only minor modifications (19). Detergent soluble proteins $(30 \mu \mathrm{g})$ were loaded and separated on a NuPAGE ${ }^{\mathrm{TM}} 4-12 \%$ Bis-Tris Gel (Invitrogen, Carlsbad, CA), electrophoresed for $40 \mathrm{~min}$ at constant $200 \mathrm{~V}$ under reducing conditions, and then transferred to a PVDF Immobilon-P Transfer Membrane (Millipore Corp., Bedford, MA) at $400 \mathrm{~mA}$ for $100 \mathrm{~min}$ in a transfer apparatus-Owl Bandit VEP-2 (Owl, Portsmouth, $\mathrm{NH}$ ) according to the manufacturer's instructions. Membranes were incubated with primary antibody overnight at $4^{\circ} \mathrm{C}$ and then with horseradish peroxidase conjugated-second antibody for $1 \mathrm{~h}$ at room temperature. The immunoreactive bands were visualized using Protein Detector TMB Western Blot Kit (KPL, Gaithersburg, MD) following the manufacturer's instructions.

\section{Results}

Preparation and $I C_{50}$ evaluation of Ppa-conjugate 2.1. The Ppa-conjugate 2.1 was prepared as described previously $(5,6)$. Preparation and $\mathrm{IC}_{50}$ evaluation were determined for both the inhibitor core $\mathbf{1 . 1}$ and the Ppa-conjugate 2.1 (Fig. 1) and revealed that both compounds were potent inhibitors of PSMA using an HPLC-based assay previously established by
A

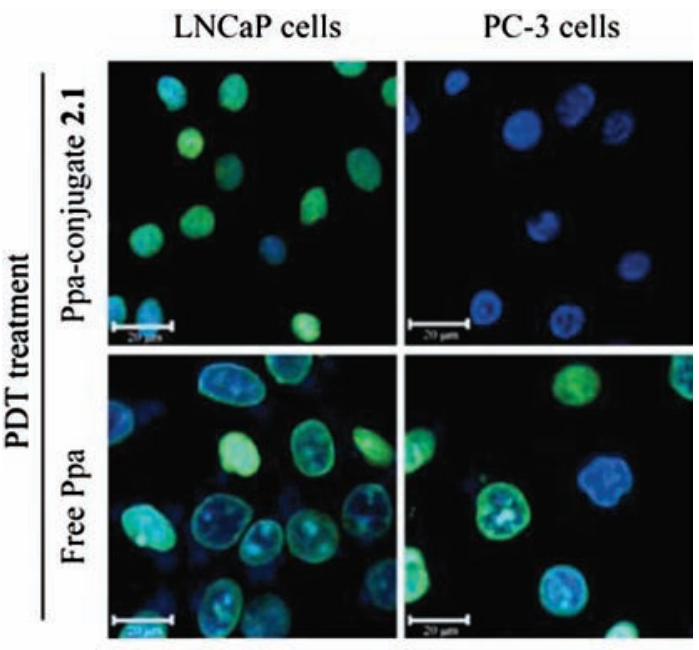

Light exposure Ppa-conjugate $\mathbf{2 . 1}$ (No inhibitor treatment) (No light exposure)

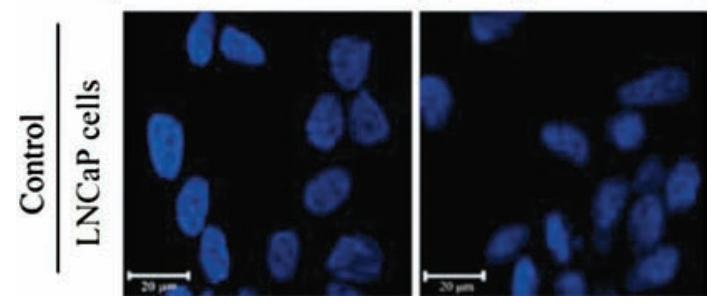

B

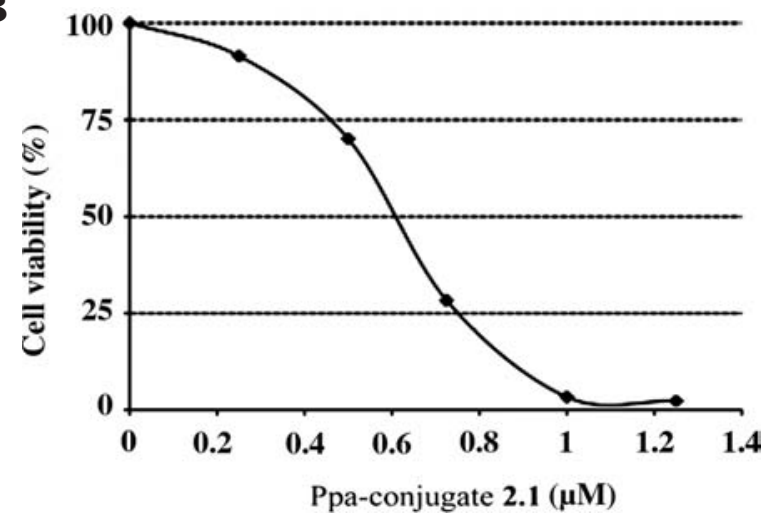

Figure 2. In vitro efficacy of targeted PDT. (A) TUNEL staining of PDTtreated cells. PDT-treated LNCaP or PC-3 cells with $1 \mu \mathrm{M}$ Ppa-conjugate 2.1. PDT-treated LNCaP and PC-3 cells with $1 \mu \mathrm{M}$ free Ppa as control. Cellular nuclei were counterstained by DAPI. Bar scale, $20 \mu \mathrm{m}$. Apoptotic nuclei are double-labeled by TUNEL (green) and DAPI (blue) while nonapoptotic nuclei are stained only by DAPI (blue). (B) Cell viability following targeted PDT. LNCaP cells were subjected to PDT with increasing concentrations of Ppa-conjugate 2.1. The cell viability was determined in triplicate using an improved MTT assay at $16 \mathrm{~h}$ post-PDT.

our group (5,16-18). The $\mathrm{IC}_{50}$ values for inhibitor core $\mathbf{1 . 1}$ and Ppa-conjugate 2.1 for PSMA were 130 and $50 \mathrm{nM}$, respectively. Ppa conjugate $\mathbf{2 . 1}$ exhibited enhanced potency against PSMA, which is a consistent phenomenon observed in our previous studies (5).

Specificity of PDT and detection of apoptotic cells by TUNEL assay. DNA fragmentation following PDT-treatment with Ppa-conjugate 2.1 was detected using the TUNEL assay (Fig. 2A). Detection of DNA fragmentation using this assay was observed as green fluorescent signals from cell nuclei as 


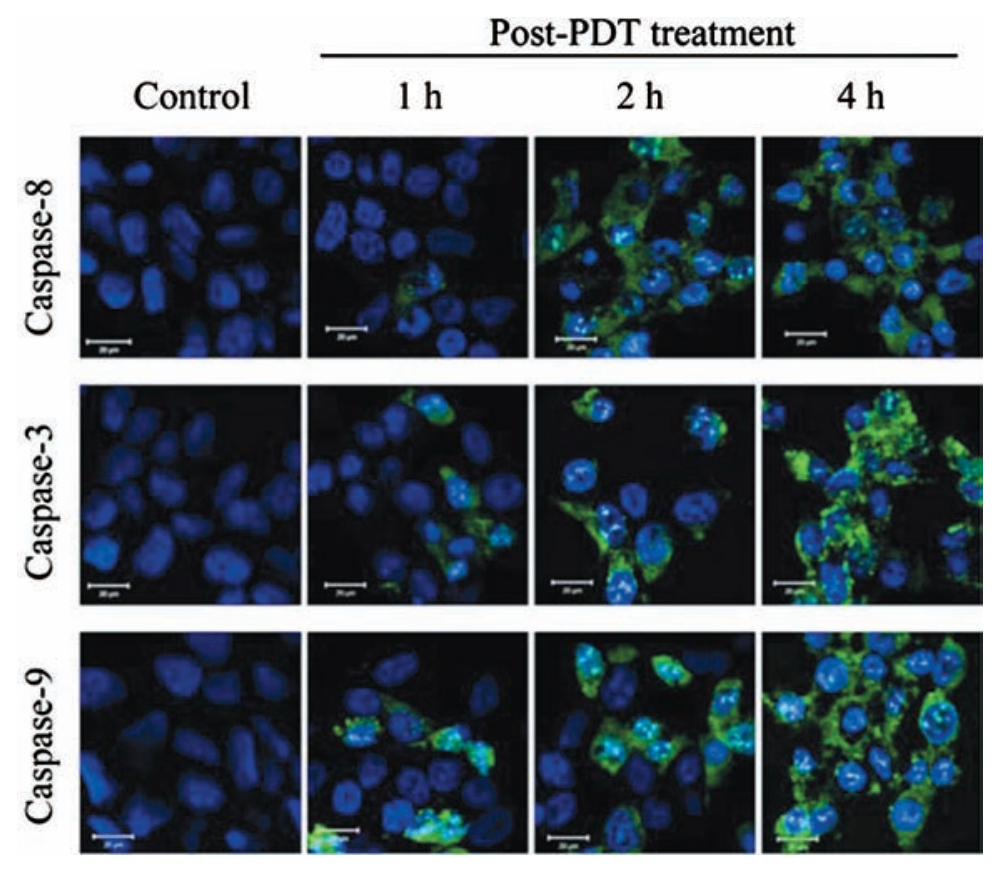

Figure 3. The detection of caspase-cascade activation after targeted PDT with Ppa-conjugate 2.1. Detection of activated caspase-8, -3 , and -9 was performed at 1, 2 and 4 h post-PDT with Ppa-conjugate 2.1 treated LNCaP cells. LNCaP cells treated with light but no Ppa-conjugate $\mathbf{2 . 1}$ served as negative controls. Activation of caspases $-8,-3$, and -9 increased from 1 to $4 \mathrm{~h}$ post-PDT. All cells were incubated with Hoechst 33342 to stain live cell nuclei (blue), prior to the final cell fixation. Ppa-conjugate $\mathbf{2 . 1}$ was administered at $1 \mu \mathrm{M}$. Bar scale, $20 \mu \mathrm{m}$.

a result of incorporation of fluorescein-dUTP into free 3'-OH terminal ends of broken single or double-strand DNAs within apoptotic cells. For these experiments, Ppa-conjugate $\mathbf{2 . 1}$ (1 $\mu \mathrm{M})$ was applied to both LNCaP $\left(\mathrm{PSMA}^{+}\right)$and PC-3 (PSMA-) cells prior to light administration. No fluorescent signal from the incorporation of fluorescein-dUTP was observed for control experiments in which LNCaP cells were exposed to either: a) Ppa-conjugate 2.1 without irradiation, or b) irradiation in the absence of Ppa-conjugate 2.1 (Fig. 2A). In contrast, PDT-treated LNCaP cells were predominately labeled with green fluorescence while no detectable incorporation of fluorescein-dUTP was observed at same concentration in the PSMA- PC-3 cells (Fig. 2A). These latter results revealed the specificity of the Ppa-conjugate 2.1 toward the PSMA ${ }^{+}$ LNCaP cells. As an additional control, unconjugated Ppa (1 $\mu \mathrm{M})$ was applied to both LNCaP and PC-3 cells. Upon light treatment of these cells, PDT-mediated DNA fragmentation was observed in all cells of both cell lines confirming the non-specificity of unconjugated Ppa (Fig. 2A). These data demonstrate that the inhibitor core $\mathbf{1 . 1}$ imparts specificity of the photosensitizer Ppa for PSMA ${ }^{+}$cells. This demonstrated specificity addresses the targeting challenge encountered by most conventional PSs.

Cytotoxicity of PDT towards LNCaP cells. The cytotoxic effect of Ppa-conjugate 2.1 for PDT-treated LNCaP cells was further assessed by an improved MTT assay at $16 \mathrm{~h}$ postPDT. The dose-response curve for cell viability (\% untreated control) is shown (Fig. 2B). Based on these experiments, the $\mathrm{LC}_{50}$ for Ppa-conjugate 2.1 was approximately $620 \mathrm{nM}$ at a constant low-intensity light dose $\left(7.5 \mathrm{~J} / \mathrm{cm}^{2}\right)$.

Activation of caspases in PDT-treated LNCaP cells. To further identify the caspase pathways associated with the targeted
PDT-mediated apoptosis in LNCaP cells, the status of caspases $-8,-9$ and -3 were determined using respective, irreversible fluorescent inhibitors of each caspase (Fig. 3). These irreversible fluorescent inhibitors readily diffuse through cell membranes, and bind irreversibly to active caspases -8 , -9 and -3 in situ within 30 min under normal cell-growth condition. The temporal and spatial distribution of the activated caspases $-8,-9$ and -3 can be clearly visualized using confocal laser scanning microscopy. It was apparent that the activation of caspases $-8,-9$ and -3 occurred at an early stage of PDTinduced apoptosis, and their activities increased in a timedependent manner over the course of $4 \mathrm{~h}$ following PDT treatment (Fig. 3).

The role and sequence of caspase activation following PDT. To address the roles of caspases $-8,-3$ and -9 in the process of targeted PDT-induced apoptosis as well as their temporal location in the caspase cascade pathway, various caspasespecific inhibitors were utilized. In situ TUNEL labeling of PDT-treated LNCaP cells pre-incubated with respective inhibitors of caspases $-8,-3$ and -9 suggested that caspase- 8 and possibly caspase- 3 play a crucial role in the initiation of the apoptotic sequence after targeted PDT with Ppaconjugate 2.1 (Fig. 4). Activation of caspases -3 and -9 was completely blocked by pre-incubation with the caspase- 8 irreversible inhibitor (Z-IETD-FMK) (Fig. 5). In contrast, Z-DEVD-FMK (caspase-3 inhibitor) or Z-LEHD-FMK (caspase-9 inhibitor) only partially blocked caspase- 8 activation (Fig. 5). These results suggest that caspase- 8 is the main initiator caspase for targeted PDT-induced apoptosis and thus responsible for the activation of downstream effectors, caspases -3 and -9 . Inhibition of caspase-9 had little effect on DNA fragmentation confirming that it is not involved in the initiation events of apoptosis. Because the 
No caspase inhibitor Caspase-8 inhibitor Caspase-3 inhibitor Caspase-9 inhibitor
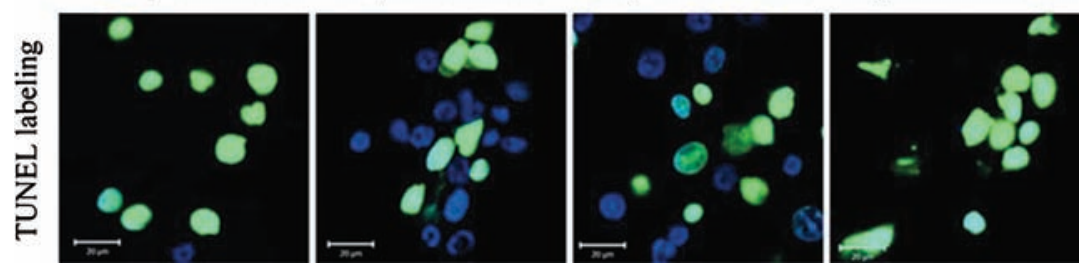

Figure 4. Protective effects on PDT-treated LNCaP cells by pre-incubation with caspase inhibitors. Pre-incubated or not LNCaP cells, were subsequently subject to targeted PDT, and TUNEL labeling at $16 \mathrm{~h}$ post-PDT. Cellular nuclei were counterstained by DAPI. Bar scale, $20 \mu \mathrm{m}$.

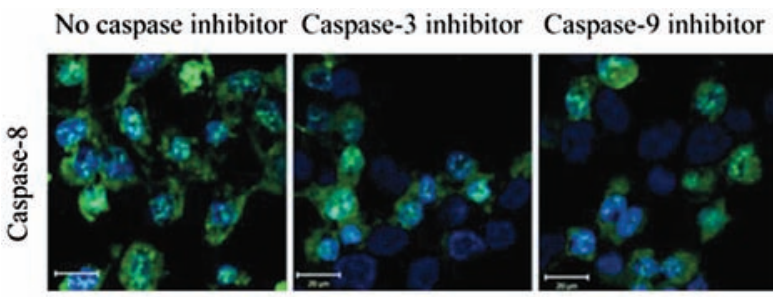

No caspase inhibitor Caspase- 8 inhibitor Caspase- 9 inhibitor
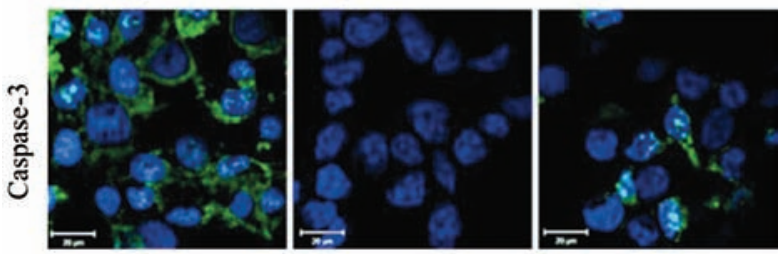

No caspase inhibitor Caspase- 8 inhibitor Caspase- 3 inhibitor
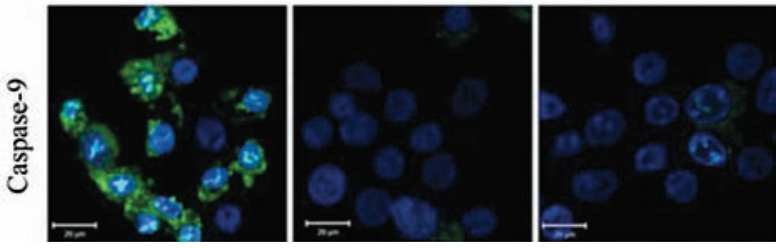

Figure 5. The sequence of activated caspases $-8,-3$, and -9 was determined by combination of fluorescent inhibitor labeling and inhibitor blocking for caspases. Cellular nuclei were counterstained by Hoechst 33342. Bar scale, $20 \mu \mathrm{m}$.

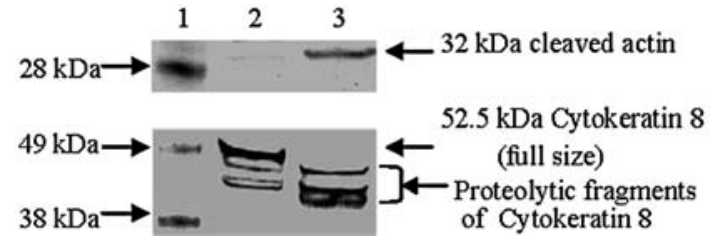

Figure 7. Targeted PDT with Ppa-conjugate 2.1 induces the cleavage of $\beta$-actin in $\mathrm{LNCaP}$ cells. Line 1, standard protein markers; line 2, the control (inhibitor treatment, without light irradiation); line 3, 2 h post-PDT. The immunoblotting of cytokeratin 8 as a control was used to verify equal loading.

inhibitors of both caspases -8 and -3 were unable to completely block DNA fragmentation within all cells, we can not exclude the activation of other caspase-independent pathways described by other researchers (20) (Fig. 4). However, our current data suggest that targeted PDT using Ppa-conjugate 2.1 induces apoptosis of LNCaP cells predominantly via the caspase-8/-3 pathway. Furthermore, PARP, a natural substrate of caspase-3, was cleaved into p85 fragment, which was detected at significant levels by $4 \mathrm{~h}$ post-PDT using a cytoimmunofluorescence method (Fig. 6). A specific 32-kDa fragment of cleaved $B$-actin by active caspase- 3 was also detected using Western blotting (Fig. 7) and cytokeratin 8 was used to verify equivalent gel-loading despite it being partially

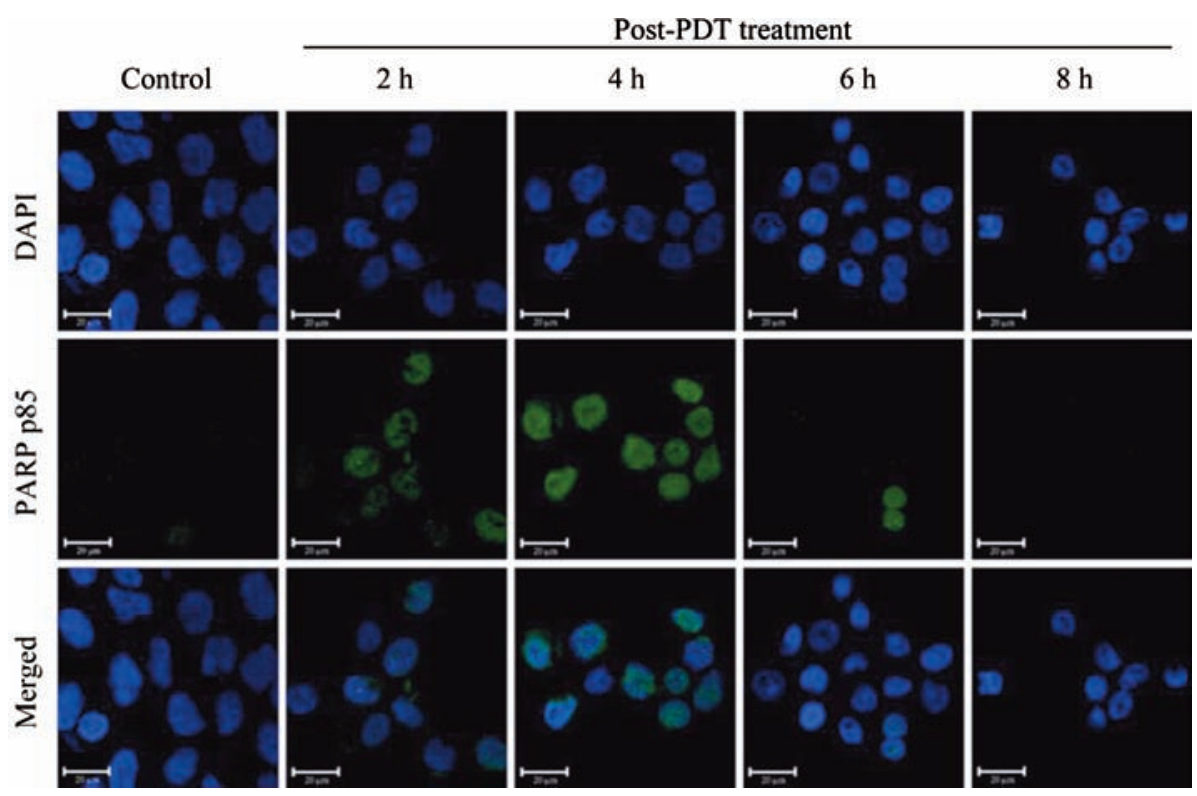

Figure 6. Detection of PARP-cleaved p85 fragment following PDT treatment. The appearance of p85 fragment in targeted PDT-treated LNCaP cells were detected immunofluorescence at 2, 4, 6 and $8 \mathrm{~h}$ post-PDT. Cellular nuclei were counterstained by DAPI. There is weak signal at $2 \mathrm{~h}$, the highest activity at $4 \mathrm{~h}$, and complete disappearance at $8 \mathrm{~h}$. In all above experiments, Ppa-conjugate 2.1 was used at $1 \mu \mathrm{M}$. Bar scale, $20 \mu \mathrm{m}$. 
proteolyzed (Fig. 7). The highly correlated temporal and spatial data for the activation of caspases -8 and -3 following the appearance of the PARP p85 fragment, cleaved $B$-actin, and morphological changes of cellular nuclei (condensed and spherical by $4 \mathrm{~h}$ post-PDT) provide further evidence of cell apoptosis via a caspase-8/-3 cascade pathway.

\section{Discussion}

Ppa-conjugate 2.1, an analog of our previous PSMA inhibitorphotosensitizer conjugate, has demonstrated a greater potency for inducing apoptosis in $\mathrm{PSMA}^{+} \mathrm{LNCaP}$ cells (6). This is correlated to its enhanced affinity for the cell surface enzymebiomarker PSMA. In comparing their chemical structures, the slightly increased-length linker between Ppa and PSMA inhibitor core may contribute to an increased flexibility between the inhibitor core and the photosensitizer thus allowing a better match to the binding surface remote from the active site of PSMA.

It is known that caspase cascade pathways are associated with biochemical and morphological changes during apoptosis (21). Two important pathways for the induction of apoptosis have been identified as 'intrinsic' and 'extrinsic'. The extrinsic pathway is related to death receptors involving caspase- 8 activation, while the intrinsic pathway is related to apoptosome complex formation involving caspase-9 activation. As initiator caspases in their respective pathways, activated caspases -8 and -9 further activate downstream effector caspases such as caspases -3 and -7 , the effect of which all leads to the late events of apoptosis. In our current study, it is clearly revealed that caspase-8, not caspase-9, plays a greater key role as an initiator in targeted PDT-induced apoptosis with Ppa-conjugate 2.1. The data from the caspase inhibition experiments suggest that while caspase-3 is downstream from the initiator effects of caspase-8, it participates in the positive feedback activation and amplification of active caspase-8. In contrast to caspase activation sequence resulting from targeted PDT with Ppaconjugate 2.1, free Ppa and its methyl ester have been reported to mediate PDT-induced apoptosis mainly via the mitochondrial caspase-9/-3 pathway in multiple cell lines $(22,23)$. This difference can partly attributed to their different sublocalization; late endosomes or lysosomes for Ppa-conjugate 2.1 and intracellular membrane systems such as mitochondria for Ppa (5,6,24-26).

Active caspases $-8,-3$ and -9 were also observed to be distributed within both nuclei and cytoplasm following targeted PDT with Ppa-conjugate 2.1. Translocation of active caspase-8 into nuclei indicates that active caspase- 8 may play a direct role on the morphological and functional changes of nuclei in addition to downstream activation of caspase-3 (27). Supporting evidence for this concept has been recently reported in which it was confirmed that activated caspase- 8 translocates to the nucleus inducing changes in lamina structure followed by spatial reorganization of telomeres and centromeres (28). Translocation of active caspase- 3 into nuclei is capable of cleaving nuclear substrates such as PARP and B-actin (29-31). The cleavage of nuclear actin leads to release of DNase I resulting in the digestion of DNA and together with the cleavage of PARP disables the repair mechanisms of the cellular response to DNA damage. Combined these processes ultimately contribute to DNA fragmentation at the late stage of apoptosis.

A question now remains as to how apoptotic initiator caspase- 8 is activated by targeted PDT with Ppa-conjugate 2.1. We have formulated two possible explanation for these results: a) induction of the extrinsic pathway including multimerization of Fas antigen (32), up-regulation expression of Fas/FasL (11) or death receptor $5(33,34)$; b) damage, proteolysis, or down-regulation, of cellular FADD-like IL-1ßconverting enzyme inhibitory protein (c-FLIP) (33). An examination of these two hypotheses is currently underway in our laboratory.

In conclusion, we have demonstrated for the first time that a photosensitizer conjugated to a small-molecule PSMA inhibitor is capable of selectively initiating apoptosis via the caspase-8/-3 pathway in $\mathrm{PMSA}^{+}$prostate tumor cells upon low-intensity light irradiation. These results are in contrast to that for free Ppa and its methyl ester, which have been reported to mediate PDT-induced apoptosis mainly via the mitochondrial caspase-9/-3 pathway in multiple cell lines. A further understanding of the cytotoxic mechanism for targeted PDT for prostate cancer is expected to guide the development of a clinically relevant treatment option for patients with primary prostate tumors in which the risk for erectile and urinary dysfunction is essentially eliminated.

\section{Acknowledgments}

This work was supported in part by the National Institutes of Health grant (7R21CA122126-03). The authors extend their gratitude for technical assistance to G. Helms and W. Hiscox at the WSU Center for NMR Spectroscopy and C. Davitt at the WSU Franceschi Microscopy and Imaging Center.

\section{References}

1. Allison RR, Downie GH, Cuenca R, Hu XH, Childs CJ and Sibata CH: Photosensitizers in clinical PDT. Photodiagnosis Photodyn Ther 1: 27-42, 2004.

2. Sharman WM, van Lier JE and Allen CM: Targeted photodynamic therapy via receptor mediated delivery systems. Adv Drug Deliv Rev 56: 53-76, 2004.

3. Detty MR, Gibson SL and Wagner SJ: Current clinical and preclinical photosensitizers for use in photodynamic therapy. J Med Chem 47: 3897-3915, 2004.

4. Berg K, Selbo PK, Weyergang A, et al: Porphyrin-related photosensitizers for cancer imaging and therapeutic applications. J Microsc 218: 133-147, 2005.

5. Liu T, Wu LY, Kazak M and Berkman CE: Cell-surface labeling and internalization by a fluorescent inhibitor of prostate-specific membrane antigen. Prostate 68: 955-964, 2008.

6. Liu T, Wu LY, Choi JK and Berkman CE: In vitro targeted photodynamic therapy with a pyropheophorbide - a conjugated inhibitor of prostate-specific membrane antigen. Prostate 69: 585-594, 2009.

7. Murphy GP, Su S, Jarisch J and Kenny GM: Serum levels of PSMA. Prostate 42: 318-319, 2000.

8. Bacich DJ, Pinto JT, Tong WP and Heston WD: Cloning, expression, genomic localization, and enzymatic activities of the mouse homolog of prostate-specific membrane antigen/ NAALADase/folate hydrolase. Mamm Genome 12: 117-123, 2001.

9. Fair WR, Israeli RS and Heston WD: Prostate-specific membrane antigen. Prostate 32: 140-148, 1997.

10. Buytaert E, Dewaele M and Agostinis P: Molecular effectors of multiple cell death pathways initiated by photodynamic therapy. Biochim Biophys Acta 1776: 86-107, 2007. 
11. Olivo M and Ali-Seyed M: Apoptosis signalling mechanisms in human cancer cells induced by Calphostin-PDT. Int J Oncol 30: 537-548, 2007.

12. Ali SM, Chee SK, Yuen GY and Olivo M: Hypericin induced death receptor-mediated apoptosis in photoactivated tumor cells. Int J Mol Med 9: 601-616, 2002.

13. Ali SM, Chee SK, Yuen GY and Olivo M: Photodynamic therapy induced Fas-mediated apoptosis in human carcinoma cells. Int $\mathbf{J}$ Mol Med 9: 257-270, 2002.

14. Moor AC: Signaling pathways in cell death and survival after photodynamic therapy. J Photochem Photobiol B 57: 1-13, 2000.

15. Morgan $\mathrm{J}$ and Oseroff AR: Mitochondria-based photodynamic anti-cancer therapy. Adv Drug Deliv Rev 49: 71-86, 2001.

16. Wu LY, Anderson MO, Toriyabe Y, et al: The molecular pruning of a phosphoramidate peptidomimetic inhibitor of prostatespecific membrane antigen. Bioorg Med Chem 15: 7434-7443, 2007.

17. Maung J, Mallari JP, Girtsman TA, et al: Probing for a hydrophobic a binding register in prostate-specific membrane antigen with phenylalkylphosphonamidates. Bioorg Med Chem 12: 4969-4979, 2004.

18. Anderson MO, Wu LY, Santiago NM, et al: Substrate specificity of prostate-specific membrane antigen. Bioorg Med Chem 15: 6678-6686, 2007.

19. Liu T, Toriyabe Y and Berkman CE: Purification of prostatespecific membrane antigen using conformational epitopespecific antibody-affinity chromatography. Protein Expr Purif 49: 251-255, 2006

20. Furre IE, Moller MT, Shahzidi S, Nesland JM and Peng Q: Involvement of both caspase-dependent and -independent pathways in apoptotic induction by hexaminolevulinate-mediated photodynamic therapy in human lymphoma cells. Apoptosis 11 : 2031-2042, 2006.

21. Agostinis P, Buytaert E, Breyssens H and Hendrickx N: Regulatory pathways in photodynamic therapy induced apoptosis. Photochem Photobiol Sci 3: 721-729, 2004.

22. Tian Y, Leung W, Yue K and Mak N: Cell death induced by MPPa-PDT in prostate carcinoma in vitro and in vivo. Biochem Biophys Res Commun 348: 413-420, 2006.

23. Matroule JY, Carthy CM, Granville DJ, Jolois O, Hunt DW and Piette J: Mechanism of colon cancer cell apoptosis mediated by pyropheophorbide-a methylester photosensitization. Oncogene 20: 4070-4084, 2001.

24. Liu T, Toriyabe Y, Kazak M and Berkman CE: Pseudoirreversible inhibition of prostate-specific membrane antigen by phosphoramidate peptidomimetics. Biochemistry 47 : 12658-12660, 2008
25. Savellano MD, Pogue BW, Hoopes PJ, Vitetta ES and Paulsen KD: Multiepitope HER2 targeting enhances photoimmunotherapy of HER2-overexpressing cancer cells with pyropheophorbide-a immunoconjugates. Cancer Res 65: 6371-6379, 2005.

26. Sun $X$ and Leung WN: Photodynamic therapy with pyropheophorbide-a methyl ester in human lung carcinoma cancer cell: efficacy, localization and apoptosis. Photochem Photobiol 75: 644-651, 2002.

27. Benchoua A, Couriaud C, Guegan C, et al: Active caspase-8 translocates into the nucleus of apoptotic cells to inactivate poly(ADP-ribose) polymerase-2. J Biol Chem 277: 34217-34222, 2002.

28. Raz V, Carlotti F, Vermolen BJ, et al: Changes in lamina structure are followed by spatial reorganization of heterochromatic regions in caspase-8-activated human mesenchymal stem cells. J Cell Sci 119: 4247-4256, 2006.

29. Decker P and Muller S: Modulating poly(ADP-ribose) polymerase activity: potential for the prevention and therapy of pathogenic situations involving DNA damage and oxidative stress. Curr Pharm Biotechnol 3: 275-283, 2002.

30. Mashima T, Naito M, Noguchi K, Miller DK, Nicholson DW and Tsuruo T: Actin cleavage by CPP-32/apopain during the development of apoptosis. Oncogene 14: 1007-1012, 1997.

31. Mashima T, Naito $M$ and Tsuruo T: Caspase-mediated cleavage of cytoskeletal actin plays a positive role in the process of morphological apoptosis. Oncogene 18: 2423-2430, 1999.

32. Takahashi H, Itoh Y, Miyauchi Y, et al: Activation of two caspase cascades, caspase-8/3/6 and caspase-9/3/6, during photodynamic therapy using a novel photosensitizer, ATX-S10(Na), in normal human keratinocytes. Arch Dermatol Res 295: 242-248, 2003.

33. Lee TJ, Um HJ, Min do S, Park JW, Choi KS and Kwon TK: Withaferin A sensitizes TRAIL-induced apoptosis through reactive oxygen species-mediated up-regulation of death receptor 5 and down-regulation of c-FLIP. Free Radic Biol Med 46: 1639-1649, 2009.

34. Schempp CM, Simon-Haarhaus B, Termeer CC and Simon JC: Hypericin photo-induced apoptosis involves the tumor necrosis factor-related apoptosis-inducing ligand (TRAIL) and activation of caspase-8. FEBS Lett 493: 26-30, 2001. 